\title{
Methodology for Measuring Phase Shifts of Signals Using Discrete Hilbert Transform
}

\author{
${ }^{1}$ Yurii Kuts, ${ }^{2,3}$ Orest Kochan, ${ }^{1}$ Iuliia Lysenko, ${ }^{3}$ Ruslana Huminilovych \\ ${ }^{1}$ Igor Sikorsky Kyiv Polytechnic Institute, Kyiv, Ukraine \\ ${ }^{2}$ School of Computer Science, Hubei University of Technology, Wuhan, China \\ ${ }^{3}$ Lviv Polytechnic National University, Lviv, Ukraine \\ Email: j.lysenko@kpi.ua
}

\begin{abstract}
The phase methods for measuring physical quantities and the phase measuring equipment are widely used in various fields of science and technology. Improving the metrological characteristics of phase measuring equipment, which is intended for use in a wide range of signal-to-noise ratio, involves the development of a methodology for phase measurements and the presentation of their results based on the harmonization of the material and physical measures of phase shifts. The paper proposes a signal processing methodology based on a combination of the discrete Hilbert transform and deterministic as well as statistical methods of phase measurement. Circular statistics such as circular mean, circular variance and the resulting vector length are proposed for use in the phase measurements.
\end{abstract}

Keywords: Signal Processing Methodology, Hilbert Transform, Circular Statistics

\section{Introduction}

The concept of the internet of things $[1,2]$ together with the progress in measurement science $[2,3]$ led to the exponential growth of measurement data. The rise of the computational power of computers created the opportunity to develop the methods for measurement data processing to improve the accuracy of measurement and their noise immunity [1-3]. This paper is devoted to the development of such a technique in phase shift measurements.

Phase methods of measuring physical quantities, transformation, and transfer of information allow effectively solving many problems in diverse fields of science and technology, e.g. in experimental physics, radiophysics, radio and radar navigation, telecommunications, geodesy, non-invasive testing, and so on. Based on the phase methods, many important scientific and technical problems related to the precise measurement of distances, time intervals, angles, analysis of the characteristics of signal fields of different physical nature have been solved.

The application of the methods required the creation of appropriate phase measuring equipment. At the initial stage of the development of the phase measuring equipment, the measurement transformation of signals' phase shifts to either the rotation angle of the electromechanical device' arm or constant voltage or time intervals was used $[4,5]$. There had already been accurate measures of voltage and time intervals that allowed simplifying the phase measuring equipment. Then later, the two-phase generators [5], which generated two coherent signals with a fixed phase shift between them, began to be used as signals' phase shift measures. That approach determined the further direction of development of the phase measurement techniques for a long time. It also determined the use of methods for processing random quantity in the algorithms of phase measurements and during the error estimation of that measurements. But the transfer of statistical analysis methods of random quantity into the field of processing the measurements of signals' phase shifts has its limitations. This is because the phase of periodic signals has a natural periodicity of $2 \pi$, which has to be taken into account when justifying the algorithms for processing phase data, and when forming the result of phase measurements and choosing the probability measure [6]. In the case of low signal-to-noise ratios in the additive mixture of harmonic signal and noise, all of these are especially important. In 
general, the abovementioned signals belong to periodic signals. The property of periodicity is manifested in the repetition of certain parts or states of processes and signals. In the case of estimating the parameters of harmonic signals, which are the components of the additive mixture with noise, the problem of processing random phase shifts occurs. Since the models of random angles are similar to the models of random phase shifts [7], the methods of statistical processing of angular data can be applied to them $[8,9]$. It should be noted that advances in computer technology and digital signal processing created the preconditions for improving the methodology of processing the phase measurements, more extensive use of complex algorithms for the statistical processing of angular data. All of these allow significantly improving the metrological characteristics of the phase measuring equipment.

The paper considers the features of the proposed methodology for processing the phase measurements based on the use of the discrete Hilbert transform (DHT) $[10,11]$ and methods of statistical processing of angular data.

\section{Basic of the Phase Measurements Methodology}

The signals can be presented as follows:

$$
u(t)=U \cos [2 \pi f t+\varphi]+n(t), u_{r}(t)=U_{r} \cos [2 \pi f t], t \in T,
$$

where $U, U_{r}$ are the trains of the signal $u(t)$ and the reference signal $u_{r}(t)$, respectively; $\varphi$ the phase shift between the harmonic signal component $u(t)$ and the reference signal $u_{r}(t) ; t, T$ are time and time interval of signal observation, respectively; $f$ is the frequency of the harmonic signals; $n(t)$ is the implementation of white noise with zero expectation and $\sigma^{2}$ variance.

There is a Fourier transform for signals from Eq. 1. After transformation to the digital signals, they are represented by samples $\{u[j], j=\overline{1, J}\},\left\{u_{r}[j], j=\overline{1, J}\right\}$, where $J=\operatorname{int}\left(T / T_{s}\right)$, where $T_{s}$ is a sampling interval. It is necessary to consider the methodology for determining the phase shift estimate based on combining the usage of the DHT and methods of statistical processing of angular data.

\section{Discrete Hilbert Transform and Its Application for Signal Phase Calculation}

The DHT and the concept of "analytical signal" introduced on its basis are widely used for theoretical researches of periodic processes and phenomena [7, 8]. The discrete analytical sequence is determined as $\dot{z}[j]=u[j]+i \tilde{u}[j]$, where $\tilde{u}[j]$ is a DTH of $u[j]$. The simplest method of DHT calculating is the spectral method. It can be found in [12]. From the definition of a discrete analytical sequence, one can find the discrete instantaneous phase of the sequence $x[n]$ :

$$
\hat{\varphi}[j]=\tan ^{-1} \frac{\tilde{u}[j]}{u[j]}+\frac{\pi}{2}\{2-\operatorname{sign} \tilde{u}[j](1+\operatorname{sign} u[j])\} .
$$

Function in Eq. 2 has a sawtooth shape and changes periodically within the range of $[0,2 \pi)$. The discrete instantaneous unwrapped phase could be obtained from Eq. 2:

$$
\hat{\Phi}[j]=\hat{\varphi}[j]+2 \pi g(\hat{\varphi}[j]),
$$

where $g(\hat{\varphi}[j])$ is a step function, which increases by one when the phase changes from $2 \pi$ to 0 .

The discrete instantaneous phase shift is determined according to the equation as follows:

$$
\hat{\varphi}_{j}=\hat{\Phi}_{j}-2 \pi f T_{s} j, j=\overline{1, J} \text {. }
$$


One can apply the methods of statistical processing of angular data to the sample from Eq. 4 and calculate several circular statistics.

\section{Circular Statistics for the Use in Phase Measurement}

The most important circular statistics in phase measurement are given in Table 1.

Table 1. Some circular statistics defined for the sample $\hat{\varphi}_{j}, j=\overline{1, J}$.

Name of circular Definition, formula

statistical characteristic

Sampling trigonometric moment of order $\mathrm{u}$ relatively to the direction $\alpha$ ( $\mathrm{u}$ is an integer)

$$
\begin{aligned}
& T_{u}(\alpha)=\frac{1}{J} \sum_{j=1}^{J} e^{i u\left(\hat{\varphi}_{j}-\alpha\right)}=a_{u}(\alpha)+i b_{u}(\alpha)=r_{u}(\alpha) e^{i m_{u}(\alpha)} \\
& a_{u}(\alpha)=\frac{1}{J} \sum_{j=1}^{J} \cos \left[u\left(\hat{\varphi}_{j}-\alpha\right)\right]=a_{u}(0) \cos (u \alpha)+b_{u}(0) \sin (u \alpha) \\
& b_{u}(\alpha)=\frac{1}{J} \sum_{j=1}^{J} \sin \left[u\left(\hat{\varphi}_{j}-\alpha\right)\right]=-a_{u}(0) \sin (u \alpha)+b_{u}(0) \cos (u \alpha)
\end{aligned}
$$

Sampling circular mean

$$
\begin{aligned}
& \varphi_{c}=\tan ^{-1} \frac{S}{C}+\frac{\pi}{2}[2-(\operatorname{sign} S) \times(1+\operatorname{sign} C)] \\
& C=\frac{1}{J} \sum_{j=1}^{J} \cos \hat{\varphi}_{j} ; \quad S=\frac{1}{J} \sum_{j=1}^{J} \sin \hat{\varphi}_{j}
\end{aligned}
$$

\begin{tabular}{ll}
\hline $\begin{array}{l}\text { Sampling length of the } \\
\text { resultant vector }\end{array}$ & $r=\sqrt{C^{2}+S^{2}}$ \\
\hline $\begin{array}{l}\text { Sampling circular } \\
\text { variance }\end{array}$ & $V=1-r$
\end{tabular}

Probability Distributions of Random Angles for the Use in Phase Measurements

The measurement result consists of one measured value and the measurement uncertainty with the stated probability of coverage [6]. The probability can be evaluated from probability density functions. The probability distribution functions of random phase shifts are the same as those of random angles. One of the characteristic features of the circle as space, on which sets of phase shifts are formed, is the periodicity (with the period $2 \pi$ ) property of the laws of the probability density distribution of random phase shifts.

The von Mises probability distribution for a random angle is determined by the formula:

$$
p_{M}(\theta \mid \mu, k)=\exp \{k \cos (\theta-\mu)\} / 2 \pi I_{0}(k),|\mu|<\infty, k>0,
$$

where $I_{0}$ is the modified Bessel function of the first kind and zero-order; $\mu$ is the circular average direction of a random angle; $k$ is the concentration parameter of a random angle in the vicinity of $\mu$.

The von Mises distribution is one-vertex and symmetric according to the value of $\mu$, which is the mathematical expectation of this distribution. Some other typical circle distributions such as uniform, triangular, wrapped normal are presented in [5]. For the wrapped normal distribution, the central limit theorem holds on the circle: for independent random angles, which have the same probability distribution function, the probability distribution of the normalized sum of angles approaches to the wrapped normal distribution [5]. An appropriate choice of the parameters of the wrapped normal distribution allows giving a reasonable approximation for it by von Mises distribution. 


\section{Using the Offered Methodology in Some Applied Problems of Phase Measurement}

The research confirms the effectiveness of the proposed methodology for phase measurements in the following applications: (i) precise phase shifts measurement when a signal-to-noise ratio close to unity; (ii) estimation of the signal period by its discrete instantaneous unwrapped phase [13]; (iii) detection of ultrasonic testing signals at a low signal-to-noise ratio using the sampling length of the resultant vector, which is calculated in the sliding mode; (iv) determination of the signal-to-noise ratio in case of the sum of the harmonic signal and Gaussian noise through sampling trigonometric moments.

\section{Conclusions}

The DHT allows determining the signal phase through the tan-1 function without a physical measure of phase. Since the signal samples are obtained with the sampling interval, it allows studying their change even within one period of the carrier signal in the case of modulation of the phase and amplitude of the harmonic signal. It also allows obtaining samples of phase shifts of large volumes to which the methods of statistical processing of angular data can be applied. Thus, it increases the measurement accuracy. The probability distribution of random phase shifts is proposed to determine the probability of coverage, coverage factor, and extended measurement uncertainty for the phase measurements.

\section{References}

[1] Jun, S., Przystupa, K., Beshley, et all. (2020). A Cost-Efficient Software Based Router and Traffic Generator for Simulation and Testing of IP Network. Electronics, 9(1), 40.

[2] Pytka, J., Budzyński, P., Józwik, J., et all. (2019). Application of GNSS/INS and an Optical Sensor for Determining Airplane Takeoff and Landing Performance on a Grassy Airfield. Sensors, 19(24), 5492.

[3] Wang, J., Kochan, O., Przystupa, K., and Su, J. (2019). Information-measuring system to study the thermocouple with controlled temperature field. Measurement Science Review, 19(4), 161169.

[4] Dorozhovets, M., Motalo, V., Stadnyk, B., Vasyliuk, V., Borek, R. and Kovalchyk, A. (2005). Fundamentals of metrology and measuring techniques, vol. 2, "Measuring techniques". Lviv Polytechnic National University Press. (In Ukrainian).

[5] Kuts, Y., Shcherbak, L. (2009). Statistical phase measurement. Ternopil Ivan Puluj National Technical University Press. (In Ukrainian).

[6] International Vocabulary of Metrology: Basic and General Concepts and Associated Terms, JCGM 200:2012 (2012).

[7] Babak, V., Yeremenko, V., Kuts, Y., Myslovych M. amd Shcherbak L. (2019). Models and measures in measurements. Naukova Dumka Publishing. (In Ukrainian).

[8] Mardia K. (2000). Directional Statistics. John Willey \& Sons.

[9] Fisher N. (2000). Statistical Analysis of Circular Data. Cambridge University Press.

[10] Bendat, J., Piersol, A. (2010). Random Data. Analysis and Measurement Procedures. John Willey \& Sons.

[11] Poularikas, A. (2010). Transforms and Applications Handbook. CRC Press LLC.

[12] Lawrence Marple, S. (1999). Computing the Discrete-Time "Analytic" Signal via FFT. IEEE Transactions on Signal Processing, 47 (9), 2600-2603.

[13] Kuts, Y., Protasov, A., Lysenko, I., Bliznuk, O. and Uchanin, V. (2017). Using multidifferential transducer for pulsed eddy current object inspection. 2017 IEEE 1st Ukraine Conference on Electrical and Computer Engineering, 826-829. 